\title{
Measurement and Analysis of the Vertical Distribution Characteristic of the Atmospheric Particle Concentration in Beijing District
}

\author{
Dawei Li and Yuening Bai
}

\begin{abstract}
In this paper, the vertical distributions of atmospheric particulate matter (PM2.5 and PM10) around residential building and in open space in Beijing district are measured and analyzed. The PM data in residential building environment is obtained by measuring it at each floor windows of the building, and the data in open space is obtained by measuring it at a rising hot-air balloon. The vertical distribution characteristics of PM concentration for different conditions of wind speed and direction are discussed. It is found that the distribution of PM concentration is in close association with the changing of wind for both residential building environment and open space. The results show that in breeze day, the highest atmospheric particle concentration around the residential building appears at a height about $20 \mathrm{~m}$, and the coverage heights (defined by PM value less than $80 \%$ of that on ground) of PM2.5 and PM10 in free space are about $400 \mathrm{~m}$ and $100 \mathrm{~m}$ respectively; but for strong windy day, the distribution of atmospheric particles tends to be smooth along the residential building, and the coverage heights of PM2.5 and PM 10 in free space are all about $300 \mathrm{~m}$. This paper also investigates the relationship between the vertical attenuation of PM concentration and the speed of wind at different heights. The natural accumulation phenomenon of atmospheric PM under the effect of microgravity is observed.
\end{abstract}

Index Terms-Air pollution, particle number concentration, PM, vertical distribution.

\section{INTRODUCTION}

The atmospheric particles matter (PM), also known as aerosols, is a result of a series of complex physical and chemical procedures [1]. The distributions of the PM in cites are now become attractive topics, and many works regarding the PM distribution measurements for both residential building environment and open space in Chinese cities, such as Shanghai, Nanjing, Beijing, Tianjin, have been done. For residential building environments, $\mathrm{Li}$ et al had measured the PM2.5 concentration distribution of the street canyon by observing at four discrete heights $(1 \mathrm{~m}, 8 \mathrm{~m}, 20 \mathrm{~m}, 38 \mathrm{~m})$ on a building facing the street canyon in Shanghai for two times [2]. Kong et al set three sampling places located respectively at heights of $1.5 \mathrm{~m}, 54 \mathrm{~m}$, and $80 \mathrm{~m}$ on a 27 floor residential building in Nanjing for observing the vertical distribution of the PM in living area in estivo-autumnal season [3], and the

Manuscript received August 9, 2013; revised September 29, 2013.

Dawei Li is with the Institute of Lightwave Technology, Beijing Jiaotong University, Beijing 100044, China (e-mail: $11111001 @$ bjtu.edu.cn).

Yuening Bai is with the Experimental High School attached to Beijing Normal University, Beijing 100032, China (e-mail: ellen.616@ hotmail.com). measurements were taken two times with 4 days in each time. These measurements were taken at a few discrete sampling points, so it can not completely reflect the vertical distribution of the atmospheric PM in residential building environment. For the open space, the measurements are mainly taken on the professional meteorological towers There are two reports concerning about the PM distribution measurement in Beijing district [4], [5]. These measurements were performed at the discrete heights on a meteorological tower with height of $325 \mathrm{~m}$ located in the Institute of Atmospheric Physics, Chinese Academy of Sciences. In the first measurement, the observing instruments was placed at heights of $8 \mathrm{~m}, 100 \mathrm{~m}$ and $320 \mathrm{~m}$ on the tower respectively; and in the second measurement, the instruments was placed at heights of $8 \mathrm{~m}, 80 \mathrm{~m}$ and $240 \mathrm{~m}$ respectively. The measurement in Tianjin was also taken on the Tianjin meteorological tower with height of $255 \mathrm{~m}$, and the device for observing PM mass concentration was placed at heights of $40 \mathrm{~m}, 120 \mathrm{~m}$ and $220 \mathrm{~m}$ on the tower respectively [6]. In this measurement, an interesting phenomenon is found: it takes about 2 hours for the fine PM to diffuse from ground into sky beyond $220 \mathrm{~m}$. Similar to those taken in the residential building environment, these measurements were only taken at 3 discrete points in the vertical height, so it cannot reflect the continuous variation of the fine particle concentration in the vertical direction. In this paper, the vertical PM concentration distributions for both the residential building environment and the open space are measured continuously. For the residential building environment, the measurement is performed using portable laser atmospheric PM concentration tester at each floor of the building; for the open space, the vertical distribution of PM concentration is measured with the help of a hot-air balloon, which is a novel and interesting trial to our knowledge.

\section{Method AND SCHEME}

\section{A. Research Scheme and Measuring Instruments}

The research of this paper is based on the realistic measurement, so the first step is measuring the distribution of concentration of the atmospheric particle matter. When the basic data is obtained, the vertical distribution property of the PM can be analyzed. Meanwhile, in order to see the reason behind the PM distribution phenomenon, the speed distribution of the airflow is simulated. In the research, the Dylos 1700 (made by BEIANG, USA) air quality monitor [7], as shown in Fig.1, is used in the measurement. It is working on the principle of laser beam scattering on the particles [8], 
and can simultaneously monitor the concentrations of the fine particle matters between 0.5-2.5 micron, and the thick particle matters beyond 2.5 micron, in real time. The fine and thick particle matters are classified as PM2.5 and PM10 respectively, which mean the total number of the corresponding particles per 0.01 cubic foot.

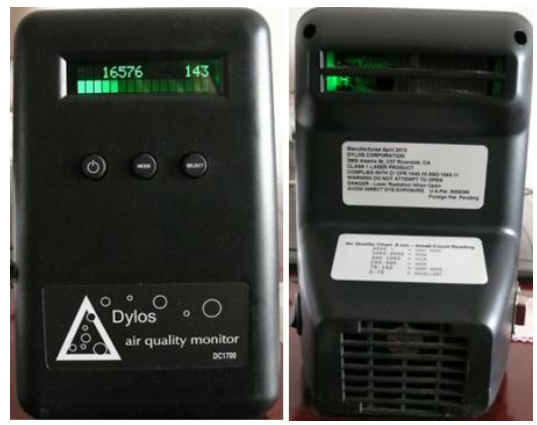

Fig. 1. Dylos1700 air quality monitor.

\section{B. Residential Building Environment and Testing Scheme}

The residential building selected to measure the PM is a 15-floor slab-type apartment building, located in the north-south direction, as shown in Fig. 2(a). In the east of the building, another 6-floor north-south direction building is located $10 \mathrm{~m}$ away. In the north of the two buildings, a $3 \mathrm{~m}$ high wall is located $10 \mathrm{~m}$ away. The western and southern sides of the buildings are the open space. The staircase of the 15-floor building is in the north side of the building with windows to the outside on each floor, as shown in Fig. 2(b), this makes convenient for testing the PM concentration. The testing scheme for the building includes following steps: 1) open all the staircase windows to mix the air inside and outside completely; 2) measure and record the PM concentration on each floor from the top of the building to the ground; 3) put the instrument outside of the staircase windows and continuously monitor the PM data at least two minutes on each floor, and then take the average and record it. There are total 17 times of testing for the whole building including on the top platform of the building and on the ground, and it takes about one hour to finish the whole work. It is also worth to mention that the data of wind direction and speed comes from the living weather report of the nearby meteorological observatory.

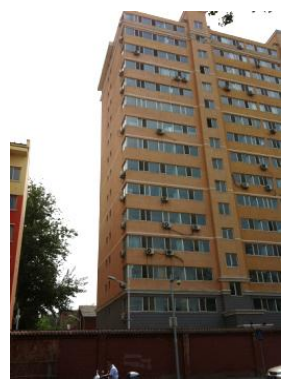

(a) Buildings

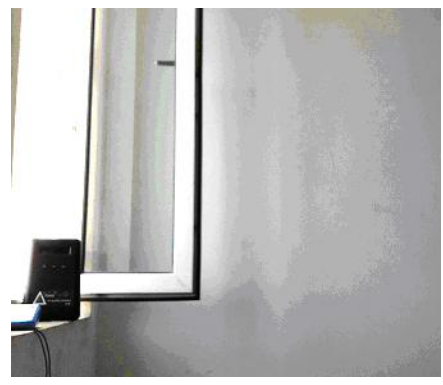

(b) Staircase windows
Fig. 2. Residential building environment.

\section{Scheme for Testing on Hot-Air Balloon}

Since the buildings block the movement of the wind, the airflow between the buildings is very complex in windy days, so the distribution of the PM concentration is different from that in open space. Only in the windless conditions, the PM concentration distribution measured on the staircase windows of building approaches that in open space. Hence, in this paper, another novel measurement of the vertical PM concentration distribution for open space is taken, that is, measuring the PM concentration continuously on a rising hot-air balloon. To our knowledge, it is the first time to measure the PM concentration in open space by the way of hot-air balloon. The location of the balloon rising is chosen in the Langfang district of Hebei province near Beijing city.

During the rising of the balloon, Dylos1700 air quality monitor is applied to measure the PM concentration continuously, and the sports barometric altimeter AVPilot made by BRAUNIGER, Germany is utilized to monitor the altitude and its changing rate. Because the balloon floating speed is synchronizing with the speed of wind, it is impossible to get the wind speed by measuring on board. Thus, we use GPS72 handheld navigator made by GARMIN, USA to measure the wind speed and direction approximately instead. The instruments are shown in Fig. 3.

The balloon is selected to take off in the time of sunrise on breeze and polluted day, because the airflow is relative stable in this period of time. The balloon flies up to $600 \mathrm{~m}$ with a uniform speed of $0.3 \mathrm{~m} / \mathrm{s}$, and stays for a while at heights of $10 \mathrm{~m}, 20 \mathrm{~m}, 30 \mathrm{~m}, 50 \mathrm{~m}, 100 \mathrm{~m}, 150 \mathrm{~m}, 200 \mathrm{~m}, 300 \mathrm{~m}, 400 \mathrm{~m}$, and $500 \mathrm{~m}$ respectively, for measuring and recording the data of PM concentration. Fig. 4 shows the preparation and taking off of the balloon.

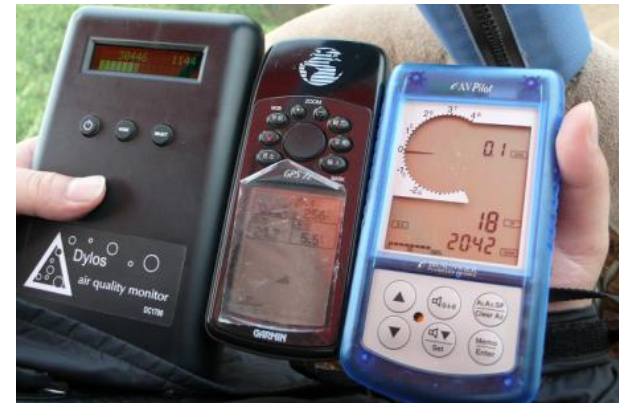

Fig. 3. Instruments for collecting and recording the data during balloon rising.

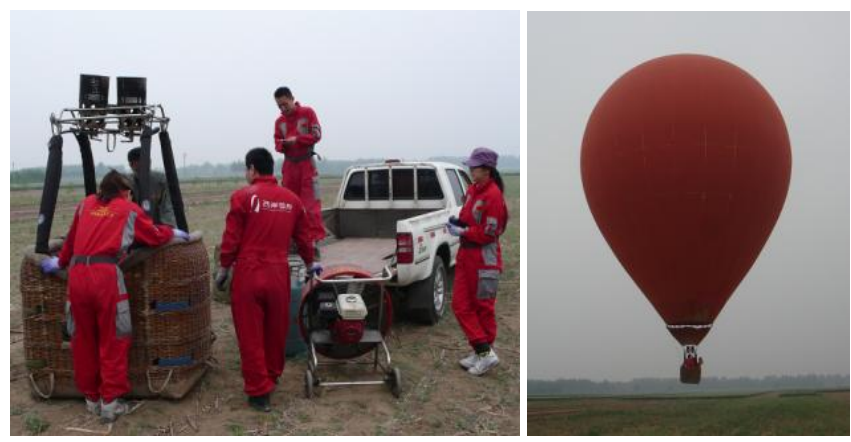

Fig. 4. Preparation and taking off of the balloon.

\section{Simulation of the Airflow around the Building}

The airflow around the residential building is simulated by software Fluent 6.3. The model of the realistic building was first established by the software Gambit. After discretizing the model, setting the corresponding boundary, and selecting the airflow surface, the final mesh model of the building was introduced into Fluent 6.3. The output numerical data of the 
Fluent 6.3 was transferred into visible speed field and pressure field by software Tecplot 360 .

\section{RESULTS AND ANALYSIS}

\section{A. PM Vertical Distribution around the Building}

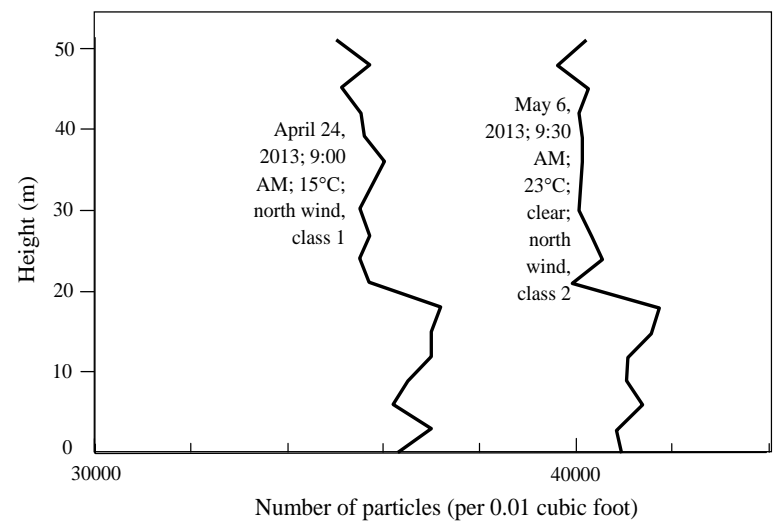

(a) Data measured on April 24 and May 6, 2013.

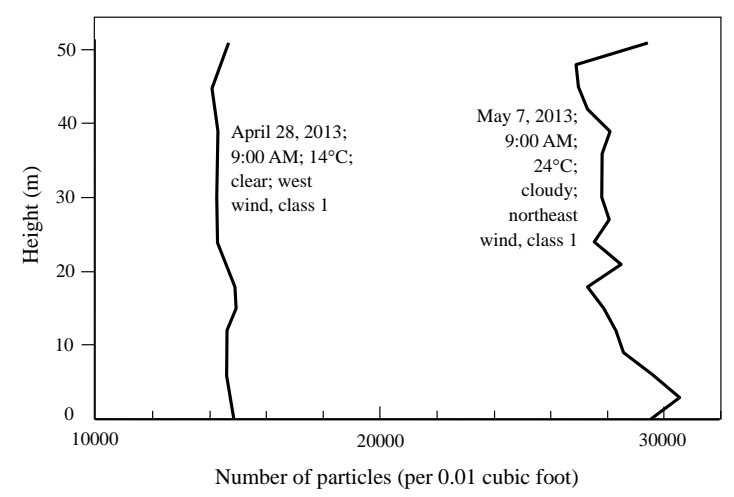

(b) Data measured on April 28 and May 7, 2013.

Fig. 5. Vertical concentration distribution of the PM 2.5 measured at the north staircase windows of the building on different days, 2013

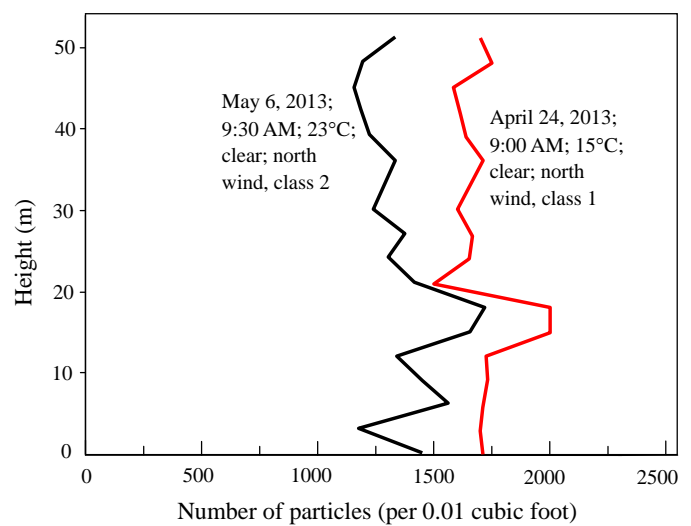

Fig. 6. Vertical concentration distribution of the PM 10 measured at the north staircase windows of the building on April 24 and May 6, 2013.

Fig. 5 gives the variations of the particle concentrations with the height of residential building ( $3 \mathrm{~m}$ for each floor) for different weather conditions and different times. Among them, April 24 and May 6 are the days with north wind, but the wind is not strong (class 1-2). Because the measurement is taken at the north staircase windows of the building, it is directly facing to the wind. April 28 is the day with west wind, so it's the side blow wind; May 7 is the day with northeast wind, so it's the oblique blow wind. April 29 is a day with strong wind blowing from the north, and the speed of wind reaches class 4-5. Fig. 6 gives the vertical concentration distribution of the PM 10 measured at staircase windows of the building on April 24 and May 6. Fig. 7 gives the results of vertical concentration distribution of both the PM2.5 and PM 10 measured on April 29, under the condition of strong wind.

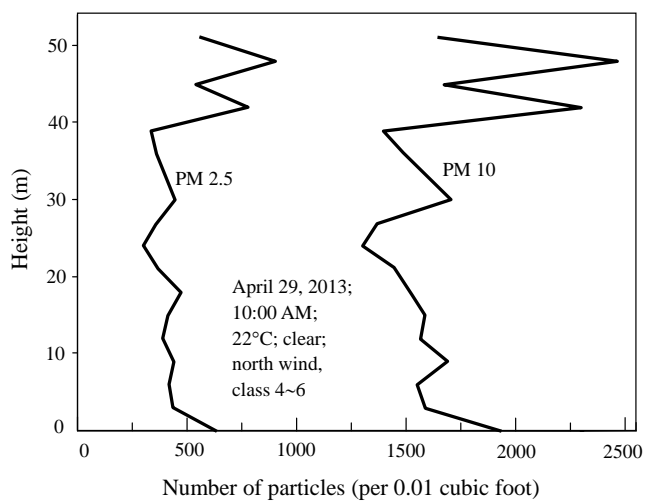

Fig. 7 Vertical concentration distribution of the PM 2.5 and PM 10 measured at the north staircase windows of the building on April 29, 2013.

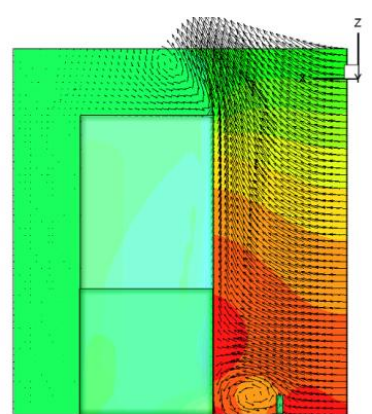

(a) Vertical plane crossing the building.

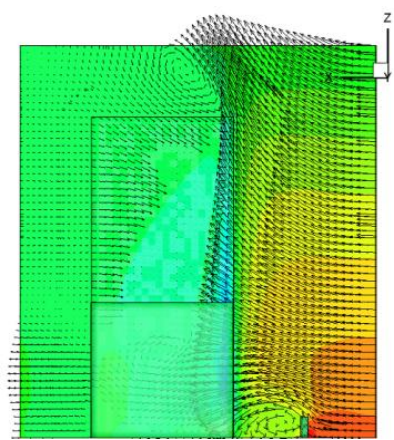

(b) Vertical plane in between two buildings.

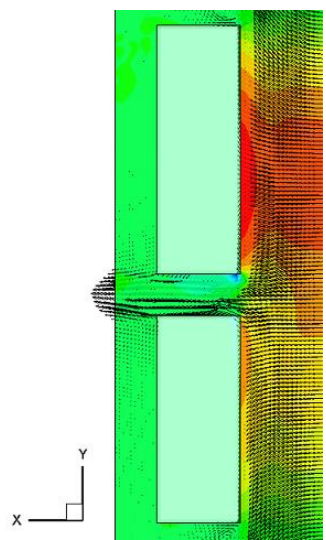

(c) Horizontal plane above the north sidewall Fig. 8. Simulated airflow around the buildings. 
Fig. 8 shows the simulation results of airflow around the building by software Fluent 6.3 , under the condition of north wind with class 2 . Fig. 8(a) shows the airflow distribution in the vertical cross section of the building passing through the testing staircase windows, and the wind is blowing within this plane. The length and the direction of the arrow in the figures represent the strength and direction of the speed of the airflow in this cross section. Fig. 8(b) shows the airflow distribution in the plane in between the 15 -floor and 6-floor buildings. Fig. 8(c) shows the airflow in a horizontal cross section above the $3 \mathrm{~m}$ high sidewall in the north of the buildings. From these figures, we can see that there are vortexes above the top platform of the building and in between the building and the north sidewall. This in some extend influences the vertical distribution of the particle concentration.

From the measured data of vertical distribution of PM concentration and the simulation airflow results, we can see that the distribution of airflow is very complex due to the complex environment around the residential buildings which can be seen in Fig. 8. Thus, the vertical distribution exhibits an irregular property around the residential buildings, which is also related to the wind direction and speed. But from Fig. 5 and Fig. 6, we can still found the essential rule that the PM concentration around the building is smaller in high altitude and larger in low altitude, especially for the case facing to the wind (wind blowing from north direction in this case). Because the wall blocks the airflow in the north side of the buildings and forms a vortex of airflow in low altitude, as shown in Fig. 8, the PM concentration in low altitude is more homogeneous and the highest value appears at a height corresponding to 6 th floor of the building. When wind blows from the side direction, the vortex vanishes, and the PM distribution in the low altitude exhibits an inhomogeneous property. However, the highest value of the PM still occurs at the height corresponding to the 6th floor. Therefore, from the measured data we can conclude that the highest PM concentration appears at the height corresponding to 6th floor (about $20 \mathrm{~m}$ in height) on breeze day in the early spring.

For the case of side blow of wind, the data measured on the top platform of building increases in a leap manner, as the curves show in Fig. 5(b). The main reason for this phenomenon is that the side blow wind creates a complex airflow field on the top platform due to the complex structure of the platform. When the wind becomes stronger, the airflow between and around the buildings will be mixed completely, so the PM concentration does not show an obvious characteristic like that occurs in the breeze day. Similarly, it slightly increases in a leap manner on the top layers, as shown in Fig.7. From the analysis we can conclude that for the strong wind day, the PM distribution rule around buildings changes little with the seasons.

\section{B. PM Distribution in Open Space Measured on Balloon}

There are total twice balloon test in our research. The first time was taken on May 5, at 6:00 AM. Before taking off, the weather report told that the AQI of PM 2.5 approaches 400, and the wind class was 1 , the pollution of air was very heavy. The realistic speeds of wind measured at $100 \mathrm{~m}, 200 \mathrm{~m}, 300$ m were class $2(6 \mathrm{~km} / \mathrm{h})$, class $3(15 \mathrm{~km} / \mathrm{h})$ and class $3(22$ $\mathrm{km} / \mathrm{h}$ ) respectively. The second time was taken on May 19, at 6:50 AM. The weather report told that the class of north wind was 2 . When take off, the speed of wind increased rapidly, and reached about class 5 at height $300 \mathrm{~m}$, and class 7 at 500 $\mathrm{m}$. Also, the direction of the wind changed with the height, as shown in Fig. 9.

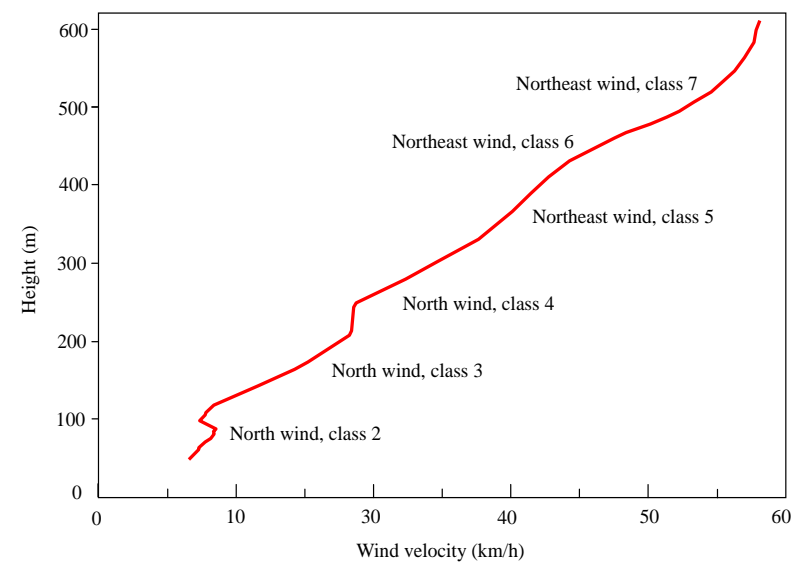

Fig. 9. Variations of wind speed and direction with the height of hot-air balloon on May 19, 2013

In the first test, due to the low speed of the wind, the PM concentration declined slowly until the height exceeds 400 $\mathrm{m}$. From ground to the height of $400 \mathrm{~m}$, the PM2.5 concentration declined only $20 \%$. But starting from $400 \mathrm{~m}$, the PM2.5 concentration declined rapidly, and reduced to $60 \%$ of that on ground at height of $500 \mathrm{~m}$. The concentrations of both the PM2.5 and PM10 declined with the height, but the decline rate of PM10 is a little bit higher, and it reached 50\% at height of $100 \mathrm{~m}$, as shown in Fig. 10. This is because that the deposition effect of larger particles is more obvious.

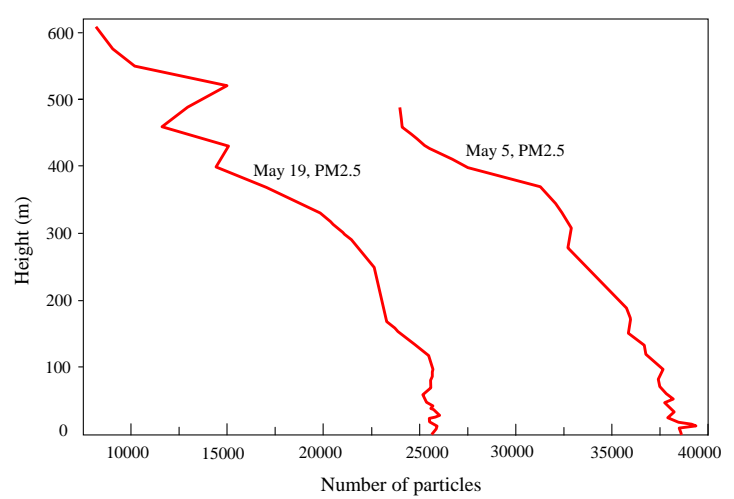

(a) The PM 2.5 measured on the hot-air balloon on May 5 and May 19, 2013

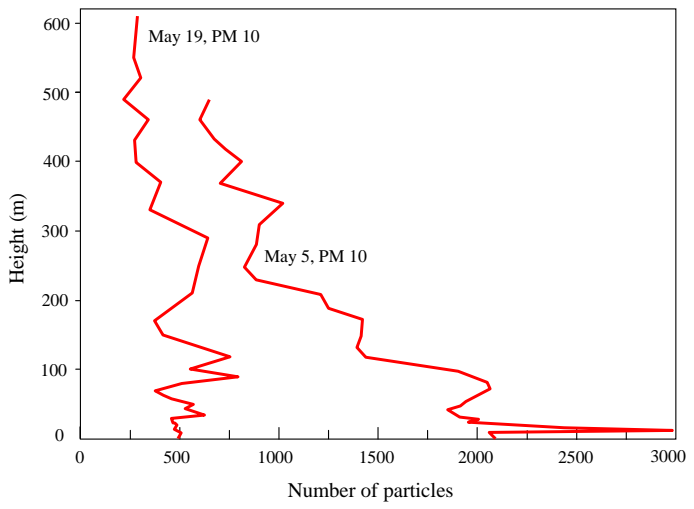

(b) The PM 10 measured on the hot-air balloon on May 5 and May 19, 2013 Fig. 10. Vertical concentration distribution of the PM2.5 and PM10. 
In the second test, the wind class reaches 7. Fig. 10 shows that the PM concentration declined faster with the height in blustery day. At $300 \mathrm{~m}$, the speed of wind reaches $30 \mathrm{~km} / \mathrm{h}$ and the PM2.5 concentration only decreases by $20 \%$. But the concentration began to decline significantly after $300 \mathrm{~m}$, and declines by $50 \%$ at $400 \mathrm{~m}$. Fluctuation appears between 400 $\mathrm{m}$ and $500 \mathrm{~m}$, within which the class of wind is between 5 and 6. At $600 \mathrm{~m}$, where the class of wind reaches to 7, the PM2.5 concentration declined to $30 \%$ of that on the ground. In this test, we found that the speed of airflow has significant effect on the PM diffusion, and this conclusion is similar to that in [9]. In addition, we found that for the stronger wind, the distribution of PM10 concentration will last for a wide vertical range in open space. As can see from Fig. 10(b), it still maintains $80 \%$ of that on the ground at height of $300 \mathrm{~m}$. The vertical distribution also fluctuates due to the strong airflow. These are the important properties of the PM10 distribution that different from those of the first test.

Comparing the data of two tests, we can find that when the wind is not strong, the cladding thickness of PM 2.5 in low atmosphere is about $400 \mathrm{~m}$ while the cladding thickness of PM10 is only about $100 \mathrm{~m}$. When the wind becomes stronger, the cladding thickness of PM 2.5 becomes thin, and is about $300 \mathrm{~m}$, while the cladding thickness of PM10 increases, and reaches to $300 \mathrm{~m}$, and the distributions of concentrations of these two particles tend to close to each other. This phenomenon reflects the effect of the wind on the diffusion of PM. And, by contrast, the distribution of PM10 is more sensitive to the change of the wind speed. This may due the reason that the strong wind compensates the gravity effect of the larger particles, and leads to the increasing of the thickness of PM10.

\section{CONCLUSION}

The research of this paper reveals that the vertical distribution of atmospheric particles in open space is different from that in the living district containing residential buildings, because the distribution of the airflow field in these two cases are in big difference. In the early spring and on the breeze day, the atmospheric particle concentration is small in the high altitude and becomes large in the low altitude for the residential building environments. The highest concentration appears at a height about twenty meters (corresponding to 6th floor). When the wind becomes stronger, the distribution of atmospheric particles tends to be smooth, because the airflow around the buildings is mixed completely. For the open space, the coverage heights (defined by height at which the PM value reduced to $80 \%$ of that on ground) of PM2.5 and PM10 are about 400 and $100 \mathrm{~m}$ respectively in the breeze pollution days; but in the strong windy and polluted days, the coverage heights of PM2.5 and PM 10 are all about 300 meters. In conclusion, the coverage height of the atmospheric particles is related to the strength of the wind in the city; when the wind is weak, the thickness of
PM 2.5 tends to higher and the thickness of PM 10 tends to lower; but when the wind gets stronger, the thickness of PM 2.5 decreases and the thickness of PM 10 increases, and at last, they tend to the same.

\section{ACKNOWLEDGMENT}

This work was done by the authors under the supervision of Professor Junhong Wang, Institute of Lightwave Technology, Beijing Jiaotong University, Beijing 100044, China.

\section{REFERENCES}

[1] J. He, G. Ma, and S. Wang, Air Pollution Control Engineering, 3rd ed. Beijing, China: Higher Education Press, 2010.

[2] X. Li, Z. Huang, J. Wang, X. Tu, and C. Yi, "Ultrafine particle number concentration and size distribution measurements in a street canyon," Environmental Science, vol. 28, no. 4, pp. 695-700, April 2007.

[3] C. Kong, S. Guo, and L. Tang, "Vertical distribution characteristics of atmospheric particles in summer and autumn in Nanjing city," Environmental Science and Management, vol. 34, no. 11, pp. 35-37, 2009.

[4] G. Ding, Z. Chen, Z. Gao, W. Yao, X. Li, X. Cheng, Z. Meng, H. Yu, J Huang, S. Wang, and Q. Miao, "Vertical structure and dynamic characteristics of lower atmosphere PM10 and PM2.5 in urban Beijing," Science in China (series D), vol. 35, pp. 31-44, 2005.

[5] W. Fan, B. Hu, and Y. Wang, "Measurements on the vertical distribution of PM2.5 concentration in fog and haze days in Beijing city," Climatic and environmental research, vol. 14, no. 6, pp. 631-638, 2009.

[6] S. Han, P. Li, and X. Li, "Vertical distribution of the $\mathrm{PM}_{2.5}$ at the ground layer in Tianjin," Ecology and Environment, vol. 17, no. 3, pp. 975-979, 2008.

[7] BEIANG, Dylos Air Quality Monitor User Manual, BEIANG, 2013.

[8] K. He, F. Yang, and F. Duan, Atmospheric Particulate Matter and Regional Compound Pollution, 1st ed. Beijing, China: Science Press, 2011, pp. 90-92.

[9] H. Min, L. He, and X. Huang, The Physical and Chemical Characteristics, Sources and Formation Mechanism of Atmospheric Fine Particles and Ultrafine Particles in Beijing, 1st ed. Beijing, China: Science Press, 2009, pp. 195-198.

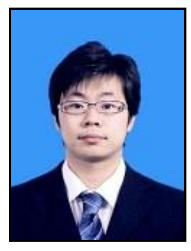

Dawei Li was born in Inner Mongolia, China. He received B.E. degree from School of Electronic and Information Engineering, Beijing Jiaotong University, Beijing, China in 2010. Currently, he is working toward the Ph.D. degree in Institute of Lightwave Technology, Beijing Jiaotong University.

His current research interests include characteristics of fluid field, sound field, electromagnetic field, and their

relationships.

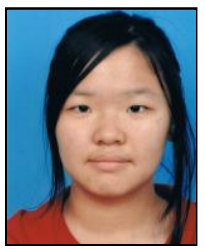

Yuening Bai was born in Beijing, China. She is a high school student in the Experimental High School Attached to Beijing Normal University. She is interested in the activities of environmental protection and is a member of extracurricular scientific group in Beijing Jiaotong University.

Ms. Bai also is a licensed pilot of hot-air balloon. 